\title{
LAMINAR DEGENERATION OF FRONTAL AND TEMPORAL CORTEX IN PARKINSON DISEASE DEMENTIA
}

\author{
Richard A. Armstrong \\ Vision Sciences, Aston University, Birmingham B4 7ET, UK; \\ Corresponding author: Dr. RA Armstrong, Vision Sciences, Aston University, \\ Birmingham B4 7ET, UK (Tel: 0121-204-4102; Fax: 0121-204-3892; Email: \\ R.A.Armstrong@aston.ac.uk)
}

Running Head: Cortical degeneration in Parkinson's disease dementia

\section{Acknowledgements}

The 'Movement Disorders Center' at Washington University School of Medicine in St. Louis is gratefully thanked for providing the PDD cases for this study. In addition, Deborah Carter and Benjamin Vincent of the Betty Martz Laboratory for Neurodegenerative Research are thanked for their expert assistance. 


\begin{abstract}
Aim: To investigate cortical laminar degeneration in Parkinson's disease (PD) with dementia (PDD).

Method: Changes in density of $\alpha$-synuclein-immunoreactive Lewy bodies (LB), Lewy neurites (LN), and Lewy grains (LG) together with surviving neurons, abnormally enlarged neurons (EN), vacuoles, and glial cell nuclei were measured across cortical laminae of frontal and temporal cortex in fifteen cases of PDD using quantitative methods and polynomial curve-fitting.

Results: Most frequently, LB and LN were distributed across all laminae while LG were distributed in upper cortical laminae. Low densities of EN were present in most cases distributed across all cortical laminae. Densities of vacuoles and glia were greatest in upper and lower cortical laminae respectively. In most gyri, there were no spatial correlations between the densities of LB, LN, and LG.

Conclusions: Cortical degeneration of frontal and temporal lobes in PDD affects all cortical laminae. Laminar distributions may result from the spread of $\alpha$-synuclein pathology from subcortical regions and subsequent spread via the cortico-cortical pathways. This spread may be a major factor in the development of dementia in PD.
\end{abstract}

Key Words: Parkinson's disease dementia (PDD); Synucleinopathy; Lewy bodies (LB); Laminar distribution; Polynomial curve fitting; Cortical degeneration 


\section{Introduction}

Parkinson's disease (PD) with dementia (PDD) is characterized by reduced gray matter volume in allocortical and neocortical regions including parietal and frontal cortices, cingulate gyrus (CG), and in temporal lobe regions such as the hippocampus and amygdala [1]. Histologically, a complex synucleinopathy is present comprising Lewy bodies (LB), Lewy neurites (LN), and Lewy grains (LG) [2,3]. The laminar distribution of a pathology in the cerebral cortex may represent degeneration of specific cortical pathways that have their cells of origin or axon terminals located within specific lamina [4]. The spread of pathology into and among cortical areas could be an important factor in the development of PDD. Hence, to investigate cortical degeneration in PDD, changes in density of the LB, LN, and LG together with abnormally enlarged neurons (EN), surviving neurons, vacuoles, and glial cell nuclei were studied across the cortical laminae in gyri of frontal and temporal lobes in fifteen cases of the disease.

\section{Materials and Methods}

\section{Cases}

PDD cases $(n=15$, mean age 74.13 years, $S D=5.74)$ (Table 1$)$ were sequentially collected autopsy cases from patients obtained from the Movement Disorders Center at Washington University School of Medicine, St. Louis, USA [5]. All patients had a clinical diagnosis of idiopathic Parkinson's disease (PD) based on modified United Kingdom Parkinson Disease Society (UKPDS) Brain Bank clinical diagnostic criteria [6]. Onset of dementia in PD was determined by clinical assessment and defined as cognitive dysfunction sufficiently severe to impair activities of daily living.

\section{Neuropathology}

All procedures were in accordance with the 1964 Declaration of Helsinki and later amendments. After death, the next-of-kin provided written consent for brain removal and retention for research studies. Brains were fixed in $10 \%$ neutral buffered formalin for at least two weeks, paraffin-embedded, and sections cut at $6 \mu \mathrm{m}$. Blocks were 
taken from the frontal lobe to study the superior frontal gyrus (SFG) (BA 8,6) and cingulate gyrus (CG) (BA 24), and from temporal lobe to study the parahippocampal gyrus (PHG) (BA 28). Immunohistochemistry was performed using phosphorylated $\alpha$-synuclein (1:10,000; Wako Chemicals USA Inc., Richmond, VA. LB stage was assessed using a PD staging scale (range: 0, 1-6), all cases being stage 5 or 6 [6] and the McKeith et al. staging scale [7,8]. Alzheimer's disease neuropathologic change (ADNC) was assessed using National Institute on Aging-Alzheimer's (NIA-AA) association guidelines 'ABC' scores [9].

\section{Morphometric methods}

The distribution of the LB, LN, LG together with surviving neurons, EN, vacuoles, and glial cell nuclei was studied from pia mater to white matter using methods described previously [10]. Five traverses from pia mater to the edge of the white matter were located randomly along each gyrus. All histological features were counted in $50 \times 250 \mu \mathrm{m}$ sample fields arranged contiguously along each traverse, the larger dimension of the field being located parallel with the surface of the pia mater. An eye-piece micrometer comprised the sample field and was moved down the traverse one step at a time from pia mater to the edge of the white matter.

\section{Data analysis}

No attempt was made to locate precisely the boundaries between individual cortical laminae. First, the degree of cortical degeneration present made laminar identification difficult. Second, identification is especially difficult in frontal cortex because it exhibits a heterotypical structure, i.e., six laminae cannot always be clearly identified and vary in prominence among cases. Third, inclusions exhibited complex patterns of distribution and did not appear to be confined to specific laminae. Hence, variations in lesion density with distance across the cortex were analyzed using a polynomial curve-fitting procedure (STATISTICA software, Statsoft Inc., 2300 East 14th St, Tulsa, OK, 74104, USA) [11]. For each gyrus, polynomials of increasing order were fitted successively to the data and the polynomial of best fit determined by an iterative process. The majority of distributions exhibited either a single density peak (unimodal) in upper or lower cortex or two density peaks (bimodal) in upper and 
lower cortex. Bimodal distributions also differed according to the relative size of the two density peaks. Spatial correlations among densities were tested using Pearson's correlation coefficient (' $r$ ').

\section{Results}

Distribution of the $\alpha$-synuclein pathology in the PHG of a case of PDD is shown in Fig 1. Microvacuolation of the superficial laminae is evident together with small numbers of LB and more abundant LN and LG.

Changes in density of the $\alpha$-synuclein-immunoreactive pathology across the cortex in a single gyrus (Case A, PHG) are shown in Fig 2. LB occurred at low density, were distributed across the cortex, and there was no significant fit to any polynomial. By contrast, the distributions of the $\mathrm{LN}(\mathrm{r}=0.87, \mathrm{P}<0.001)$ and $\mathrm{LG}(\mathrm{r}=0.90, \mathrm{P}<0.001)$ were both fitted by fourth-order polynomials suggesting bimodal distributions, the larger density peak being located in upper cortex.

In all gyri and cases (Table 2), in 26/39 (67\%) gyri there were no significant changes in density of LB across the cortex. However, LB were distributed in lower laminae in $10 / 39$ (26\%) gyri, while in further three gyri, the distribution was bimodal, densities being greater in lower cortex. LN were evenly distributed across the cortex in 22/44 (50\%) gyri and LG in 6/44 (14\%) gyri. In 14/44 (32\%) and 28/44 (64\%) gyri respectively, LN and LG exhibited a bimodal distribution with a larger upper density peak. In 8/44 (18\%) gyri, surviving neurons exhibited a single density peak in upper cortex while in 13/44 (30\%) gyri, the distribution was bimodal with a larger upper density peak. EN showed no significant change in density across the cortex, 29/32 (91\%) gyri exhibiting this distribution. In 25/44 (63\%) gyri, the greatest density of the vacuolation occurred either in upper cortex or was bimodal, the greater density peak occurring in upper cortex. Glial cell nuclei were more abundant in lower cortical laminae.

Significant positive correlations between LN and LG were observed in 14/44 (32\%) of gyri, greatest densities being present in upper cortex (Table 3). The densities of surviving neurons and vacuoles were positively correlated in 16/44 (36\%) gyri. 


\section{Discussion}

The most consistent patterns of laminar distribution in PDD were: (1) vacuolation of superficial laminae and (2) increasing gliosis with distance from pia mater to white matter. The $\alpha$-synuclein-immunoreactive pathology was frequently distributed across all laminae but where a more localized distribution was present, LG occurred in upper laminae, LB in lower laminae, and LN were more variable. The laminar distribution of the LB in PDD was similar to that reported previously in dementia with Lewy bodies (DLB) indicating their pathological similarity [4].

In normal elderly brain, neurons in frontal and temporal cortex often exhibit a bimodal distribution, the upper density peak (corresponding to laminae II/III neurons) being larger than the lower (corresponding to laminae V/VI neurons) [12]. In PDD, a similar distribution was observed in $6 / 44$ gyri, while in a further seven gyri, the distribution was bimodal, densities being similar in upper and lower cortex. In 23 gyri, there were no significant changes in density across the cortex. These results suggest significant neuronal loss affecting upper laminae, a region containing the cells of origin of the feedforward cortico-cortical projections, frequently compromised in $\mathrm{AD}$ [13] and frontotemporal lobar degeneration (FTLD) [14]. However, gliosis and LB pathology also suggest neuronal loss in lower cortical laminae potentially compromising the feedback cortico-cortical [13] and cortical/subcortical projections.

The densities of LN and LG were spatially correlated in about a third of gyri but neither were spatially correlated with LB. Hence, LB occur in cell bodies in lower laminae while LN and LG may result from neuritic and synaptic degeneration of the same or connected neurons in laminae II/III. In addition, vacuole and surviving neuron densities were positively correlated in a third of gyri. In sporadic CJD (sCJD), vacuoles are clustered around neurons [15] while in the cerebellum of variant CJD (vCJD), clusters of vacuoles in the molecular layer are negatively correlated with surviving Purkinje cells [16]. Hence, vacuolation of superficial cortical laminae in PDD could be the result of the degeneration of ascending cortical projections and loss of laminae II/III neurons. 
Hence in PDD, the pathology may spread into the cerebral cortex from subcortical areas initially affecting lower cortical laminae and resulting in LB and a significant gliosis. Subsequent spread via the ascending projections to upper cortical laminae results in the formation of LN and LG. Degeneration of upper cortical neurons results in microvacuolation of superficial laminae. Finally, the pathology spreads among gyri via the feedforward and feedback cortico-cortical pathways [13]. Variations in laminar distribution among gyri and cases could reflect different stages of this process. Spread of $\alpha$-synuclein pathology into the cortex and among cortical areas could be an important factor in the development of dementia in PD [17].

\section{Conflict of interest}

The author declares no conflict of interest.

\section{References}

[1]. Melzer TR, Watts R, MacAskill MR et al (2012) Grey matter atrophy in cognitively impaired Parkinson disease. J Neurol Neurosurg Psychiatr 83: 188-194

[2]. Saito Y, Kawashima A, Ruberu NN et al (2003) Accumulation of phosphorylated $\alpha$-synuclein in aging human brain. J Neuropath Exp Neurol 62: 644-654

[3]. Armstrong RA, Kotzbauer PT, Perlmutter JS, Campbell MC, Hurth KM, Schmidt

RE, Cairns NJ (2014) A quantitative study of $\alpha$-synuclein pathology in fifteen cases of dementia associated with Parkinson disease. J Neural Transm 121: 171-181.

[4]. Armstrong RA, Cairns NJ, Lantos PL (1997) Laminar distribution of cortical Lewy bodies and neurofibrillary tangles in dementia with Lewy bodies. Neurosci Res Commun 21: 145-152.

[5]. Kotzbauer PT, Cairns NJ, Campbell MC, Racette BA, Tabbal SD, Perlmutter JS (2012) Pathological accumulation of $\alpha$-synuclein and $A \beta$ in Parkinson disease patients with dementia. Arch Neurol 23: 1-6. 
[6]. Braak H, Ghebremedhin E, Rub U, Bratzke H, Del Tredici K (2004) Stages in the development of Parkinson disease-related pathology. Cell Tissue Res 318: 121-134

[7]. McKeith IG, Galasko D, Kosaka K et al (1996) Consensus guidelines for the clinical and pathologic diagnosis of dementia with Lewy bodies (DLB): report of the consortium on DLB international workshop. Neurology 47: 1113-1124

[8]. McKeith IG, Dickson DW, Lowe J et al (2005) Diagnosis and management of dementia with Lewy bodies: third report of the DLB Consortium. Neurology 65: 1863-1872

[9]. Hyman BT, Phelps CH, Beach TG et al (2012) National Institute on AgingAlzheimer's Association guidelines for the neuropathologic assessment of Alzheimer's disease. Alz \& Dement 8: 1-13.

[10]. Duyckaerts C, Hauw JJ, Bastenaire F, Piette F, Poulain C, Rainsard V, JavoyAgid F, Berthaux P (1986) Laminar distribution of neocortical senile plaques in senile dementia of the Alzheimer type. Acta Neuropathol 70: 249-256.

[11]. Armstrong RA, Hilton AC (2011) Statistical Analysis in Microbiology: Statnotes. Wiley Blackwell, Hoboken, NewJersey.

[12]. Armstrong RA, Slaven A (1994) Does the neurodegeneration of Alzheimer's disease spread between visual cortical regions B17 and B18 via the feedforward or feedback short cortico-cortical projections? Neurodegeneration 3: 191-196.

[13]. De Lacoste M, White CL (1993) The role of cortical connectivity in Alzheimer's disease pathogenesis: a review and model system. Neurobiol Aging 14: 1-16.

[14]. Armstrong RA, Ellis W, Hamilton RL et al (2010) Neuropathological heterogeneity in frontotemporal lobar degeneration with TDP-43 proteinopathy: a quantitative study of 94 cases using principal components analysis. J Neural Transm. 117: $227-239$. 
[15]. Armstrong RA, Lantos PL, Cairns NJ (2001) Spatial correlations between the vacuolation, prion protein deposits, and surviving neurons in the cerebral cortex in sporadic Creutzfeldt-Jakob disease. Neuropathology 21: 266-271

[16]. Armstrong RA, Ironside J, Lantos PL, Cairns NJ (2009) A quantitative study of the pathological changes in the cerebellum of 15 cases of variant Creutzfeldt-Jakob disease. Neuropathol Appl Neurobiol 35: 36-45

[17]. Steiner JA, Angot E, Brunden P (2011) A deadly spread: cellular mechanisms of $\alpha$-synuclein transfer. Cell Death and Differ 18: 1425-1433 
Table I. Demographic features, duration of disease, Lewy body (LB) stage and National Institute on Aging-Alzheimer's (NIA-AA) ABC scores rating the degree of Alzheimer's disease neuropathological change (ADNC) of the PDD cases.

\begin{tabular}{llllllll}
\hline Case & Sex & $\underline{\text { Age }}$ & $\underline{\text { Duration }}$ & $\underline{\text { LB }}$ & & \multicolumn{2}{l}{ ADNC (NIA-AA) } \\
& & & & $\underline{\text { stage }}$ & $\underline{A}$ & $\underline{B}$ & $\underline{C}$ \\
A & F & 82 & 9 & 6 & 0 & 2 & 0 \\
B & M & 80 & 7 & 6 & 3 & 2 & 0 \\
C & M & 78 & 12 & 6 & 1 & 3 & 0 \\
D & M & 79 & 6 & 6 & 1 & 1 & 0 \\
E & M & 71 & 8 & 6 & 3 & 2 & 0 \\
F & M & 78 & 6 & 6 & 3 & 3 & 0 \\
G & M & 76 & 6 & 6 & 3 & 1 & 0 \\
H & M & 67 & 4 & 6 & 3 & 1 & 0 \\
I & M & 67 & 3 & 6 & 3 & 1 & 0 \\
J & F & 73 & 7 & 6 & 0 & 1 & 0 \\
K & M & 76 & 1 & 6 & 3 & 3 & 0 \\
L & M & 77 & 11 & 6 & 1 & 1 & 0 \\
M & M & 64 & 1 & 5 & 3 & 1 & 0 \\
N & F & 66 & 13 & 6 & 0 & 1 & 0 \\
O & M & 78 & 6 & 6 & 0 & 2 & 3 \\
\hline & & & & & & & \\
\hline
\end{tabular}

Abbreviations: $\mathrm{M}=$ Male, $\mathrm{F}=$ Female 
Table 2. Frequencies of laminar distributions exhibited by the histological features across the cortex in frontal and temporal lobe in 15 cases of Parkinson's disease dementia (PDD). Where a bimodal distribution is present, data indicate the relative size of density peaks in upper and lower cortex. NS = no significant change in density across cortex.

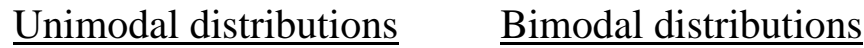

\begin{tabular}{|c|c|c|c|c|c|c|c|}
\hline$\underline{\text { Feature }}$ & $\begin{array}{l}\text { Upper } \\
\text { cortex }\end{array}$ & $\begin{array}{l}\underline{\text { Middle }} \\
\text { cortex }\end{array}$ & $\begin{array}{l}\text { Lower } \\
\text { cortex }\end{array}$ & $\begin{array}{l}\text { Upper > } \\
\underline{\text { lower }}\end{array}$ & $\begin{array}{l}\text { Upper }= \\
\underline{\text { lower }}\end{array}$ & $\begin{array}{l}\text { Upper < } \\
\underline{\text { lower }}\end{array}$ & $\underline{\mathrm{NS}}$ \\
\hline Lewy bodies & es 0 & 0 & 10 & 0 & 0 & 3 & 26 \\
\hline $\begin{array}{l}\text { Lewy } \\
\text { neurites }\end{array}$ & 3 & 0 & 5 & 8 & 4 & 2 & 22 \\
\hline Lewy grains & s 5 & 1 & 4 & 15 & 6 & 7 & 6 \\
\hline $\begin{array}{l}\text { Enlarged } \\
\text { neurons }\end{array}$ & 1 & 0 & 2 & 0 & 0 & 0 & 29 \\
\hline Neurons & 8 & 0 & 0 & 6 & 7 & 0 & 23 \\
\hline Vacuoles & 18 & 1 & 3 & 7 & 5 & 1 & 9 \\
\hline Glia & 0 & 1 & 39 & 0 & 3 & 0 & 1 \\
\hline
\end{tabular}


Table 3. Frequency of spatial correlations (Pearson's ' $r$ ') between histological features $(\mathrm{LB}=$ Lewy bodies, $\mathrm{LN}=$ Lewy neurites, $\mathrm{LG}=$ Lewy grains, $\mathrm{EN}=$ Abnormally enlarged neurons, $\mathrm{V}=$ Vacuolation, $\mathrm{SN}=$ Surviving neurons, GL $=$ Glial cell nuclei) across cortical gyri in 15 cases of Parkinson's disease dementia (PDD). NS $=$ no significant correlation .

\section{Frequency of correlation}

\begin{tabular}{|c|c|c|c|}
\hline$\underline{\text { Correlated variables }}$ & $\underline{+r}$ & $\underline{-r}$ & $\underline{\mathrm{NS}}$ \\
\hline $\mathrm{LB} / \mathrm{LN}$ & 3 & 2 & 40 \\
\hline $\mathrm{LB} / \mathrm{LG}$ & 1 & 2 & 42 \\
\hline $\mathrm{LB} / \mathrm{EN}$ & 3 & 0 & 43 \\
\hline $\mathrm{LB} / \mathrm{N}$ & 1 & 0 & 45 \\
\hline $\mathrm{LB} / \mathrm{V}$ & 1 & 1 & 44 \\
\hline $\mathrm{LB} / \mathrm{GL}$ & 3 & 0 & 43 \\
\hline $\mathrm{LN} / \mathrm{LG}$ & 14 & 1 & 29 \\
\hline LN/EN & 0 & 0 & 45 \\
\hline $\mathrm{LN} / \mathrm{N}$ & 4 & 2 & 39 \\
\hline $\mathrm{LN} / \mathrm{V}$ & 4 & 3 & 38 \\
\hline LN/GL & 1 & 5 & 39 \\
\hline LG/EN & 2 & 0 & 42 \\
\hline $\mathrm{LG} / \mathrm{N}$ & 4 & 2 & 39 \\
\hline LG/V & 4 & 7 & 36 \\
\hline LG/GL & 9 & 8 & 30 \\
\hline $\mathrm{EN} / \mathrm{N}$ & 0 & 1 & 42 \\
\hline EN/V & 2 & 2 & 41 \\
\hline EN/GL & 5 & 1 & 37 \\
\hline $\mathrm{N} / \mathrm{V}$ & 16 & 2 & 37 \\
\hline $\mathrm{N} / \mathrm{G}$ & 1 & 3 & 41 \\
\hline V/GL & 7 & 8 & 30 \\
\hline
\end{tabular}




\section{Legends to figures}

Fig 1. Distribution of the pathology in the upper cortex of the parahippocampal gyrus (PHG) in a case of Parkinson's disease dementia (PDD). The microvacuolation of superficial laminae is evident together with small numbers of LB (arrowhead) and more extensive development of LN (star) and LG (arrow). ( $\alpha$-synuclein immunohistochemistry, haematoxylin, bar $=50 \mu \mathrm{m}$ )

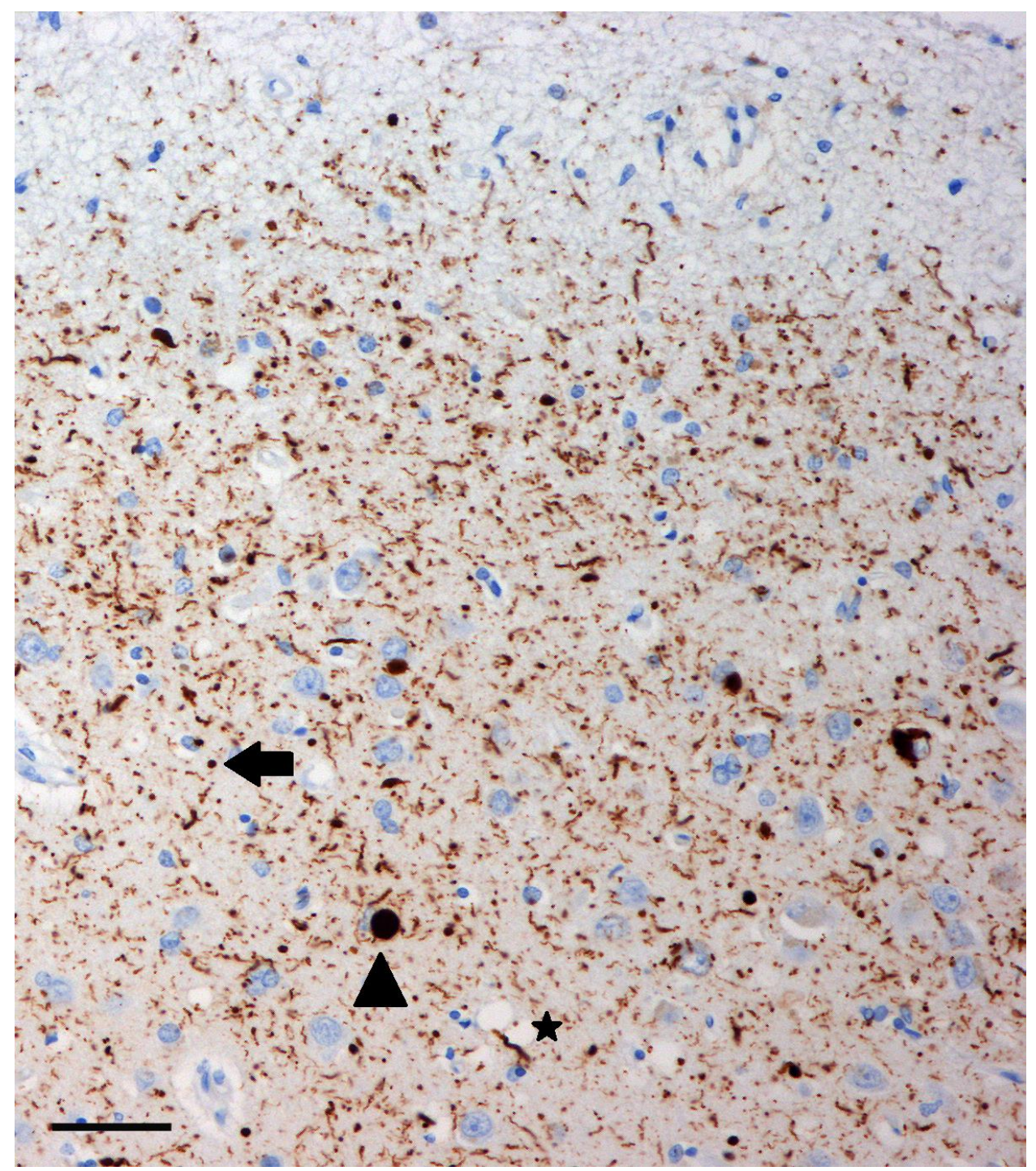


Fig 2. Examples of the distribution of the $\alpha$-synuclein-immunoreactive pathology in the parahippocampal gyrus (PHG) in a case of Parkinson's disease dementia (PDD). Curves of best fit: LB no significant polynomial, $\mathrm{LN}$ fourth-order polynomial $(\mathrm{r}=$ 0.87, $\mathrm{P}<0.001) ; \mathrm{LG}$, fourth-order polynomial $(\mathrm{r}=0.90, \mathrm{P}<0.001)$.

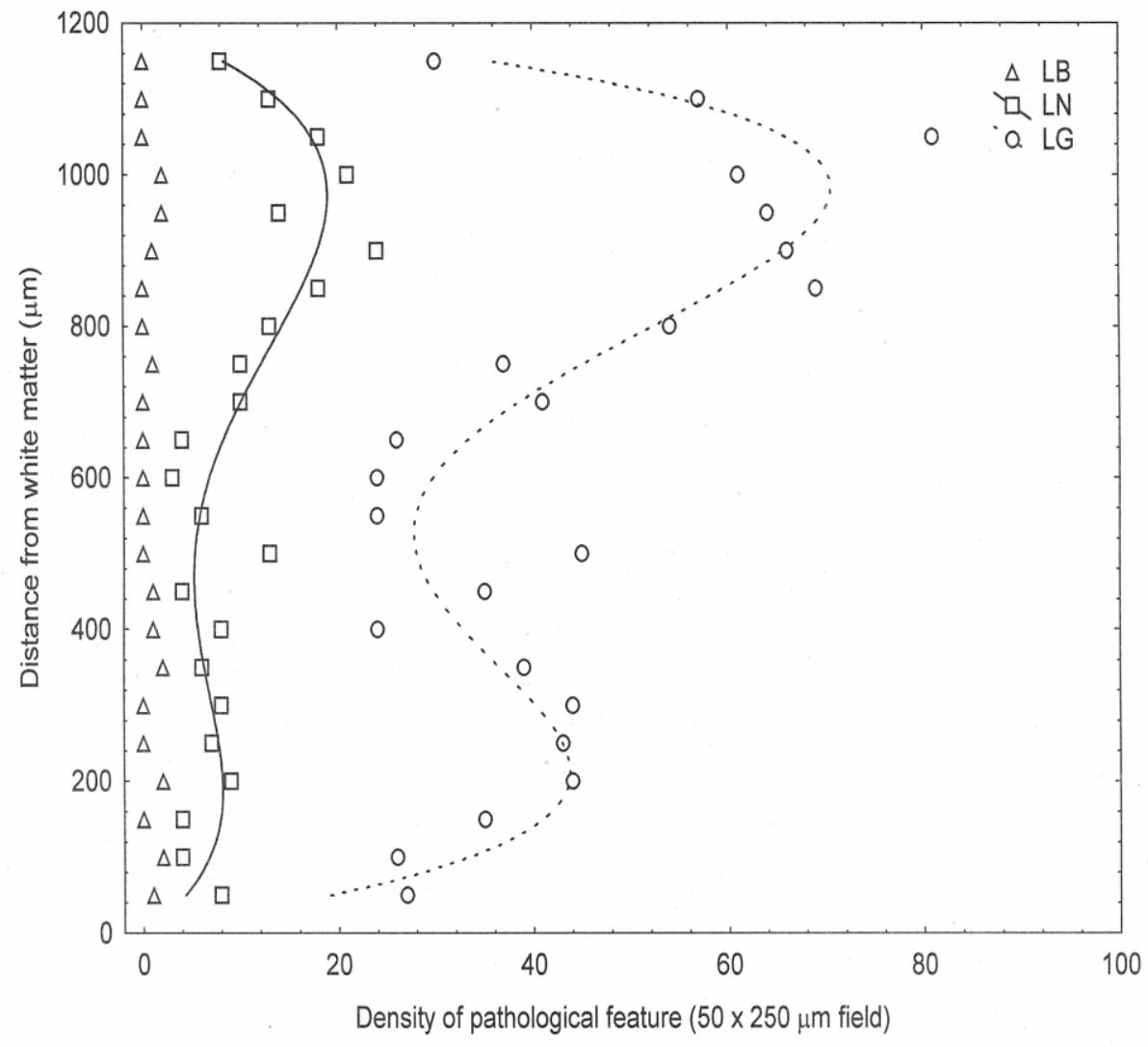

